\title{
Blockchain Based Smart Auction Mechanism for Distributed Peer-to-Peer Energy Trading
}

\author{
Md. Ezazul Islam, Madhu Chetty, Suryani Lim, Mehmood Chadhar, and Syed Islam \\ Federation University Australia \\ e.islam, madhu.chetty, suryani.lim, m.chadhar, and s.islam@federation.edu.au
}

\begin{abstract}
Blockchain based framework provides data immutability in a distributed network. In this paper, we investigate the application of blockchain for peer-to-peer $(P 2 P)$ energy trading. Traditional energy trading systems use simple passing mechanisms and basic pricing methods, thus adversely affect the efficiency and buyers' social welfare. We propose a blockchain based energy trading mechanism that uses smart passing of unspent auction reservations to (a) minimise the time taken to settle an auction (convergence time), (b) maximise the number of auction settlement; and (c) incorporate second-price auction pricing to maximise buyers' social welfare in a distributed double auction environment. The entire mechanism is implemented within Hyperledger Fabric, an open-source blockchain framework, to manage the data and provide smart contracts. Experiments show that our approach minimises the convergence time, maximises the number of auction settlement, and increases the social welfare of buyers compared to existing methods.
\end{abstract}

\section{Introduction}

Blockchain provides a data structure and an operating environment which ensure the immutability of distributed ledgers over a distributed network [1]. The data structure is made up of timestamped blocks interlinked using cryptographic hash functions, thus enabling audit trails including detection of data tampering [2] [3] [4]. Smart contracts, computer scripts within the distributed system, append new data in the blockchain. Valid users execute these smart contracts to conduct all functionality related to data read and write: they are the interface between peers and blockchain ledgers [5]. Smart contracts enable decentralisation because there is no central point of execution and this is a departure from traditional database systems [6]; therefore removing the intermediaries, reducing broker fees [7] and market settlement cost [8]. A decentralised system is more vulnerable to security threats but the blockchain data structure and smart contracts provide in-built security measures [9] [10].

Due to the distributed nature of blockchain technology and geographic dispersion of business activities; blockchain has gained attention from various sectors such as supply chain [11], electric vehicle operations [12], and energy trading [13] [14] [15]. Thakur et al. [15] showed how peer based local double auction can enhance social welfare by passing unspent auction reservations to the neighbour peer. However, the passing mechanism is naive as it overlooks the further auction capability of neighbour auctioneer, it has negative impact on efficiency (convergence time and number auction settlement) and buyers' social welfare. We call the peers as auctioneers who settle local auction. Some of the challenges within the energy trading environment include but not limited to pricing, transparency, efficiency of the auction mechanism, and social welfare of both buyers and sellers. The objective of this paper is to (a) minimise the convergence time, (b) maximise the number of auction settlements, and (c) improve buyers' social welfare in a distributed double auction. In a double auction: both buyers and sellers propose a set of prices being distributed, and several auctioneers conduct the auctions. To understand how this works, we first describe how blockchain fits in a distributed double auction environment.

Figure 1 shows how blockchain fits in a distributed double auction environment. This trading environment has buyers $\left(B_{1}, B_{2} \ldots B_{\mathrm{n}}\right)$, sellers $\left(S_{1}, S_{2} \ldots S_{\mathrm{n}}\right)$, and auctioneers $\left(A_{1}, A_{2} \ldots A_{\mathrm{n}}\right)$. Note that buyers, sellers and auctioneers are peers in a blockchain ecosystem and they communicate with the blockchain network through smart contracts for all buying/selling reservations and auctioning. All auction logics and data access requests are processed through smart contracts in this distributed system.

Energy trading in smart grids is an application that requires decentralisation in a distributed secure 


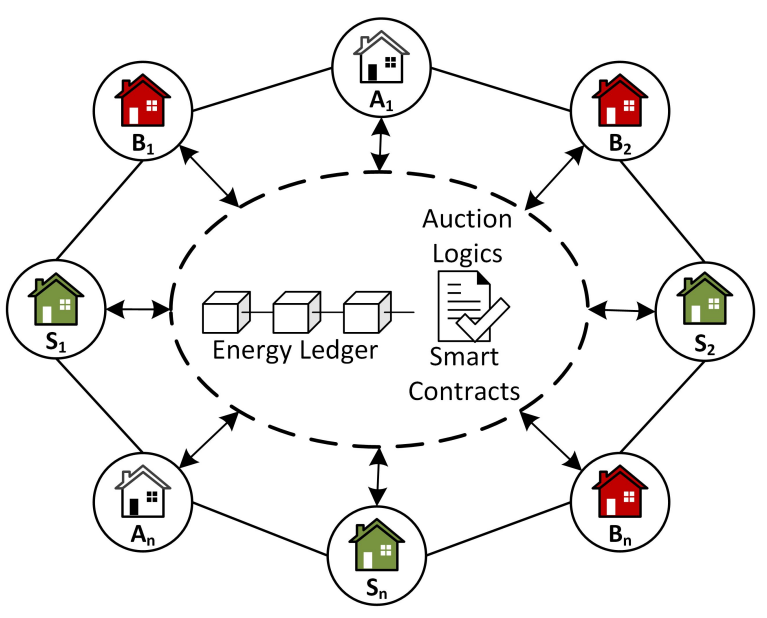

Figure 1. Blockchain in a double auction setting, which comprises buyers $\left(B_{1}, B_{2} \ldots B_{\mathbf{n}}\right)$, sellers $\left(S_{1}, S_{2} \ldots S_{\mathbf{n}}\right)$, and auctioneers $\left(A_{1}, A_{2} \ldots A_{\mathbf{n}}\right)$

environment. Peer based energy trading facilitates distributed operation of network to better utilize grid assets and energy resources [1]. The rise in the renewable energy sector (such as rooftop solar panels) turns consumers into producers, collectively known as prosumers [8]. As such, it makes sense to support these prosumers so they can trade with other prosumers within the smart grids: this is a departure from current practice which allows prosumers to trade with only a central energy provider. To shift from a central trading, we need a mechanism to support pricing and auction settlement in distributed peer-to-peer energy trading. The pricing will affect the social welfare of prosumers and the auction settlement will affect the efficiency of the energy trading process.

In this paper, we propose a blockchain based distributed double auction model to (a) minimise the convergence time (time taken to settle an auction), (b) maximise the number of auctions settlement, and (c) maximise buyers' social welfare. The blockchain framework provides the backbone of the entire peer-to-peer (P2P) energy network using smart contracts as an interface between peers and data (blockchain ledgers). Using Hyperledger Fabric, an open-source blockchain platform [16], the entire mechanism is implemented to manage the data and provide smart contracts. Both buyers and sellers are peers and some of the peers become auctioneer to contribute to the energy trading.

We organise the rest of the paper as follows. In Section 2, we provide relevant background information on double auction and second-price auction. Then in Section 3, we introduce our proposed model. After that, in Section 4, we describe the experimental setup of the proposed model. Then we describe the experiment results and analysis in Section 5. The paper concludes with a direction for future work in Section 6.

\section{Traditional Energy Trading Mechanisms}

The most straightforward auction process is the first-price auction, where buyers propose buying prices and the buyer with the highest bid wins: the auction price is the highest price. A variation of this is the second-price auction, where the winner of an auction pays the second highest price [17] [18]. An alternative to the highest bidding pricing method is second-price auction: the bidder who bids the highest amount wins the auction but pays at the second-highest price [17] [18]. As buyers pay less than the highest bidding price, they reap more benefit (profit) than that of the first-price auction. This profit contributes to social welfare of buyers, which is measured in terms of utility. If a buyer bids at price $b$ and pays $p$, then the utility is $b-p$ : the buyer tends to pay less than the bidding price [19]. In the second-price auction method, if there is no second bidder in the auction then the auction may not settle. To avoid this situation, Chen et al. [20] introduced a bid floor which is set by the auction authority and we used as a second price when there is no second bidder.

The auctioning process becomes more complicated in the double auction, where buyers propose buying prices and sellers propose selling prices [21]. This is more complex as the auction price depends not only on the proposed buying prices but also on the proposed selling prices. The following section describes the process of finding an auction price in a double auction.

We can settle a double auction if and when it reaches an equilibrium point [15] [22] :

- Sort the buying reservation prices $R\left(B_{\mathrm{i}=1 \ldots \mathrm{N}}\right)$ in descending order

- Sort the selling reservation prices $R\left(S_{\mathrm{i}=1 \ldots \mathrm{N}}\right)$ in ascending order

- The equilibrium point is when $R\left(B_{\mathrm{i}}\right)<=R\left(S_{\mathrm{i}}\right)$

Figure 2 shows double auction price determination and total utility of buyers. In addition, it shows the utility gain when we incorporate second-price method on double auction for price determination; we discuss this situation in next section. In the same figure, $R\left(B_{\mathrm{i}}\right)$ is the buying reservation curve which has a down-word slope and $R\left(S_{\mathrm{i}}\right)$ is the reservations from the sellers which has an up-word slope. The equilibrium price is where these two curves intersect and at this point 


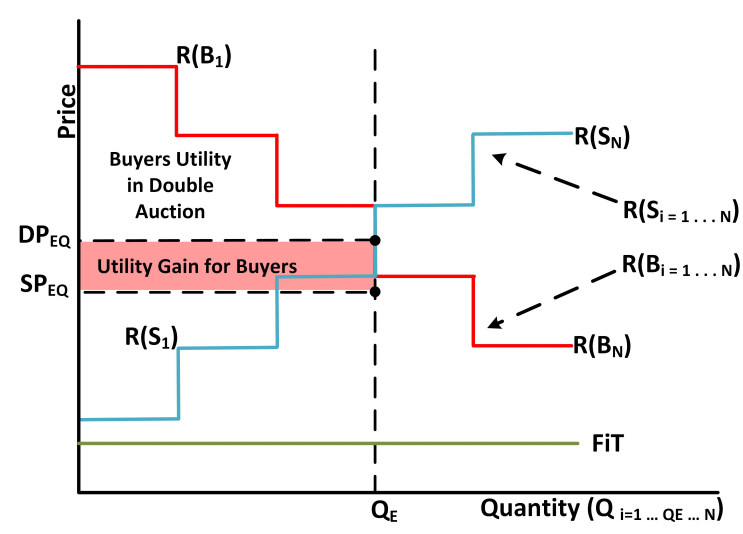

Figure 2. Buyers utility when using double auction for auction price determination and utility gain for buyers when using second-price on double auction for price determination

$R\left(B_{\mathrm{i}}\right)<=R\left(S_{\mathrm{i}}\right)$. We mark it as $D P_{\mathrm{EQ}}$. As they intersect at $D P_{\mathrm{EQ}}, \mathrm{Q}_{\mathrm{E}}$ becomes equilibrium quantity. So, $Q_{\mathrm{E}}$ number of energy units will be sold at $D P_{\mathrm{EQ}}$ price. $D P_{\mathrm{EQ}}$ is the auction price (equilibrium price); where $i=1 \ldots Q_{\mathrm{E}}$ and we use following equation to find this equilibrium price in double auction.

$$
D P_{\mathrm{EQ}}=\left(R\left(B_{\mathrm{i}}\right)+R\left(S_{\mathrm{i}}\right)\right) / 2
$$

At this price $\left(\mathrm{DP}_{\mathrm{EQ}}\right)$ we calculate buyers utility benefit, which represents social welfare. In the energy market, the maximisation of social welfare plays a vital role [23]. Maximising buyers' social welfare attracts more buyers and hopefully more sellers within a microgrid. Total utility of buyers in double auction becomes:

$$
T U\left(B D_{\mathrm{DA}}\right)=\sum_{i=1}^{Q_{\mathrm{E}}}\left(\left(R(B i)-D P_{\mathrm{EQ}}\right) Q_{\mathrm{i}}\right)
$$

Some researchers tried to maximise social welfare in distributed energy trading, such as Thakur et al. [15] and Majumder et al. [24]. Hassija et al. [7] and Tushar et al. [21] introduced another pricing method; which is peer based local double auction through smart contracts in distributed setup. This gives more social welfare to prosumers. Further enhancement by incorporating second-price auction method on double auction method is possible as it can increase social welfare of buyers. To the best of our knowledge, there has been no work that incorporates second-price auction method into a double auction method.

In the next section, we describe how the double auction fits in the blockchain environment.

\section{Proposed Blockchain Based Energy Trading Mechanism}

In a traditional environment, one central auctioneer processes all buying and selling reservations. However, in the context of energy trading in microgrids, having multiple auctioneers has the following advantages:

- Reduction in the total convergence time (the time taken to settle an auction) because multiple auctions can coincide [15].

- Reduction in electricity transmission cost because physically close peers trade with each other [7] [15] [8].

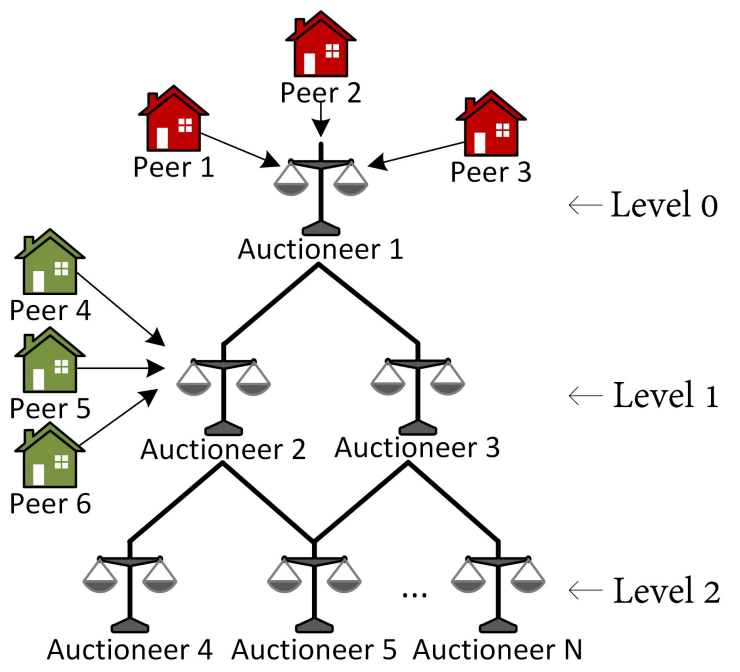

Figure 3. Levels in Peer-to-Peer Microgrid Neighbourhood

When a trading environment has multiple auctioneers, the auctioning process becomes distributed, hence the name distributed double auction. A distributed double auction introduces another complexity. In a centralised double auction, the auctioneer has all the buying and selling reservations, but in a distributed scenario, an auctioneer may have only a subset of these reservations. As such, the auctioneers may not be able to satisfy all reservations in the entire trading ecosystem. One method to overcome this issue is to pass reservations that could not be settled to other auctioneers. We call such reservations as unspent reservations. According to Figure 3, immediate neighbours of Auctioneer 1 are Auctioneer 2 and Auctioneer 3. Peer 1 to 3 are closest to Auctioneer 1 (Level 0) and are further away from Auctioneer 2 and Auctioneer 3 (Level 1). 
In a recently reported work by Thakur et al. [15], an auctioneer passes any unspent reservations to its immediate neighbours, hoping that they will be able to conduct an auction. Further, in [15], they used the double auction to minimise the overall convergence time without considering buyers' social welfare. They also passed unspent auction reservations to an immediate neighbour without considering the possibility of future auction capability of the immediate neighbour and this may adversely impact the overall convergence time.

In our proposed model, we combine the second-price auction method to maximise buyers' social welfare and using a bid floor (acceptable minimum price) to protect seller's social welfare. In other words; the bid floor can contribute to ensuring the smart grid trading system is financially sustainable. Maximising buyers' social welfare may encourage more buyers in the market, but as buyers have the potential to be sellers as well, indirectly we may have more sellers entering the market. To maximise buyers' social welfare as well as minimising the overall convergence time, we introduced two changes: (1) incorporate second-price auction method into distributed double auction mechanism to maximise the social welfare of the buyers, (2) provide asynchronous auctions with smart passing of unspent auction reservations to reduce the overall convergence time. We also use blockchain to manage data and provide smart contracts for delivering all services.

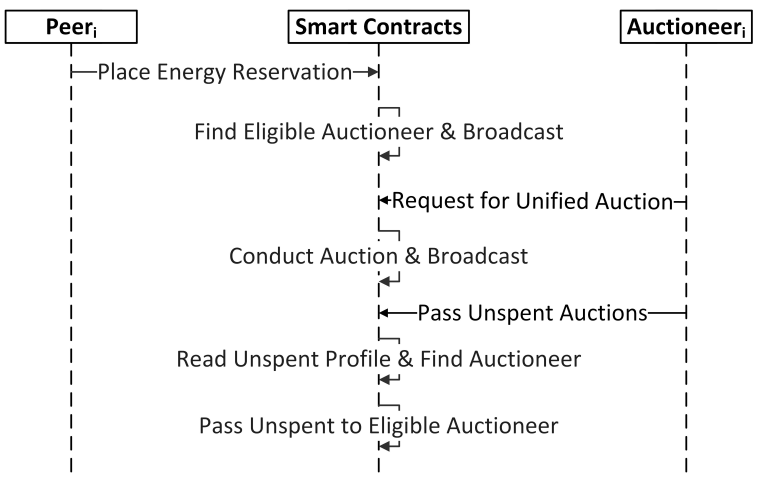

Figure 4. Operations of the Proposed Model in Blockchain Environment

As auction reservations from energy generating and producing houses will be generated based on their energy requirement, there will be multiple auction reservations for each auction settlement throughout the microgrid. Figure 4 shows all the necessary activities from a peer and an auctioneer. These activities are executed through smart contracts: Peer $_{i}$ generates a new energy reservation for buying or selling using smart contracts and Auctioneer ${ }_{i}$ passes unspent reservations and conducts the auctions via smart contracts. The following sub-sections describe our proposed model in more detail. Following sub-section describes the trading mechanism, including smart passing of unspent auction reservations and the incorporation of the second-price method into blockchain based distributed double auction. The sub-section after the following one describes the design aspects of blockchain.

\subsection{Trading Mechanism}

As shown in Figure 4, a peer within the network can make buying or selling reservation requests by executing a smart contract. Smart contract gives the initial submission to the auctioneer closest to the requesting peer. The selected auctioneer conducts the auction if it has matching auction reservation requests or passes the request to other auctioneers. The mechanism for passing the requests is central to minimising the convergence time, while second-price method will improve buyers' social welfare. This sub-section includes novel approaches for smart passing of unspent auction reservations and second-price auction in blockchain based distributed double auction.

3.1.1. Smart Passing of Unspent Auction Reservations. To facilitate smart passing of unspent auction reservations, our blockchain based distributed double auction model maintains the information about the neighbourhood auctioneer data and unspent reservation profile of all auctioneers as two matrices.

- Neighbourhood auctioneer data: This data provides the information on who the neighbouring auctioneers are and used to find neighbour auctioneers [15]. The neighbourhood data are stored in an undirected graph (implemented as a 2D matrix) stored as a block in our simulation's Hyperledger Fabric. We denote this graph as peer connection profile. Note that each auctioneer may receive reservation requests from multiple peers as shown in Figure 3. According to Thakur et al. [15] if the peers in Level 0 generates energy reservations, then an auctioneer can be selected only from the neighbours among the same or next level as depicted in Figure 3.

- Unspent reservation profile of all auctioneer peers: The unspent reservation profile (URP) stores the number of unspent auction reservations allocated for any auctioneer for a certain period of time, as seen in Figure 5 and $A_{1}, A_{2} \ldots A_{5}$ are the auctioneers. Before Time $3, A_{4}$ has -1 (buying) unspent auction reservation, so it needs 1 (selling) 
unspent auction reservation for an auction to settle. On the other hand, at the same time, $A_{1}$ has 1 as unspent reservation (one unspent selling reservation) so it can satisfy one buyer. $A_{2}, A_{3}$ and $A_{5}$ have no unspent reservation, but $A_{3}$ can settle two auctions and $A_{5}$ is capable of settling three auctions.

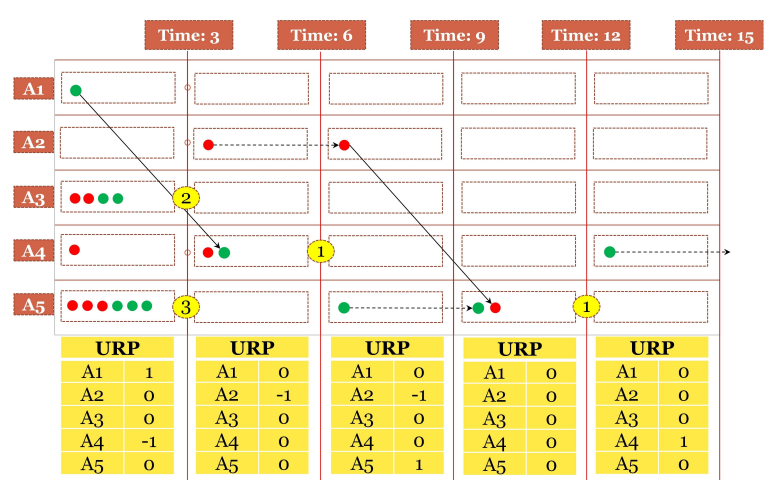

Figure 5. Smart Passing of Unspent Auction Reservations

The processes for smart passing are as follow. The passing rule selects the most capable neighbouring auctioneer that has matching unspent reservations. Figure 5 depicts the passing process. Red dots denote auction reservations from buyers, green dots from sellers. The numbers in ellipses refer to the number of auctions settled at times mentioned. Solid lines with arrows represent the smart passing of unspent auction reservations when no auction is settled. Dashed lines with arrows indicate the unspent reservations stay with the same auctioneer.

Smart contract in blockchain decides auction capability based on the URP matrix. In Figure 5, $A_{1}$ and $A_{4}$ each have one unspent auction reservation before Time 3. In this case, $A_{1}$ can pass the unspent auction reservation to $A_{4}$ or vice versa. The auctioneer that will pass is randomly selected. The same figure shows that at Time $3 A_{1}$ passes to $A_{4}$. The smart contract updates this matrix, so the URP for $A_{1}$ and $A_{4}$ are now 0 immediately after Time 3: $A_{4}$ can now settle an auction. As in [15], we limit the number of passes of an auction reservation using a trail number and this number is incremented by one for each pass. This is to ensure that trading should take place with physically close peers. The trail number represents convergence time [15]. Between Time 3 and 6, $A_{4}$ has two auction reservations (one red dot and one green dot), so smart contract settles one auction on behalf of $A_{4}$ at Time 6 (no more dots and one yellow ellipse to indicate one successful auction). The passing mechanism is the same for $A_{2}$ when it has 1 buying reservation. Between Time 12 and 15, $A_{4}$ receives one selling request. As no other auctioneer available in the neighbourhood that has at least one buying unspent reservation, $A_{4}$ cannot settle this auction: Time 15 is the expiration time of the auction, so it can no longer pass this request.

The auction mechanism will determine the winners in the auction and the buying/selling price as explained in the following section.

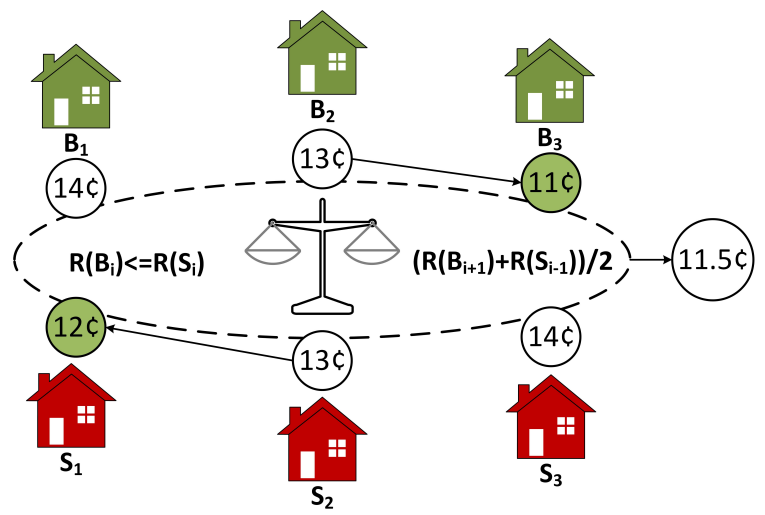

Figure 6. Incorporating second-price method into double auction. The equilibrium price is $13 c$, the average price of proposed buying and selling prices when $R\left(B_{\mathbf{i}}\right)<=R\left(S_{\mathbf{i}}\right)$. The average of the second-prices $\left(R\left(B_{\mathbf{i}+\mathbf{1}}\right)+R\left(S_{\mathbf{i}-\mathbf{1}}\right)\right) / 2$ is $\mathbf{1 1 . 5}$

Recall that in a double auction, both the buyers and sellers propose buying and selling prices. We incorporate second-price method in the double auction method. Traditionally the auction price is the equilibrium price in a double auction. However, in our proposed model, we determine the auction price by applying second price method on double auction equilibrium price. The following paragraphs describe how we incorporate the second-price auction method into a double auction method.

3.1.2. Second-price in Double Auction. Using Figure 6 as an example, the equilibrium price is $13 c$ in double auction, but the auction price is $11.5 \mathrm{c}$ when we incorporate second-price auction method on double auction equilibrium price. This is because $R\left(B_{\mathrm{i}+1}\right)$ is $11 c$ and $R\left(S_{\mathrm{i}-1}\right)$ is $12 \mathrm{c}$, and hence the average price is $11.5 c$. Although any peer can sell or buy electricity, only auctioneers can conduct the auction or pass unspent auction reservations (the auctioneers are pre-selected peers within the blockchain network).

Figure 2 depicts that: when we incorporate second-price method on double auction method, the equilibrium price curve shifts to $S P_{\mathrm{EQ}}$ point. Equation 1 shows; to choose double auction price we use $i_{\text {th }}$ 
price for both buyers and sellers. To determine auction price using second-price method by considering double auction, we pick $(i+1)$ th price from buyers reservations and $(i-1)_{\text {th }}$ price from sellers reservations. For both parties, it leads to a lower price. This shifts the auction price equilibrium from $D P_{\mathrm{EQ}}$ to $S P_{\mathrm{EQ}}$; where $i=1 \ldots Q_{\mathrm{E}}$ and we use following equation to find equilibrium price by incorporating second-price auction on double auction.

$$
S P_{\mathrm{EQ}}=\left(R\left(B_{\mathrm{i}+1}\right)+R\left(S_{\mathrm{i}-1}\right)\right) / 2
$$

Due to the shift of equilibrium price (from $\mathrm{DP}_{\mathrm{EQ}}$ to $\mathrm{SP}_{\mathrm{EQ}}$ ), buyers gain additional utility. We formulate this utility gain as:

$$
\Delta U=\left(D P_{\mathrm{EQ}}-S P_{\mathrm{EQ}}\right) Q_{\mathrm{E}}
$$

So, upon incorporation of second-price on double auction method, total utility of buyers becomes:

When total utility of buyers in double auction is: $T U\left(B D_{\mathrm{DA}}\right)$ and total utility of buyers is: $T U\left(B_{\mathrm{SP}}\right)$ if we incorporate second-price on double auction method. Our simulation and results section shows that: $T U\left(B D_{\mathrm{DA}}\right)<T U\left(B_{\mathrm{SP}}\right)$.

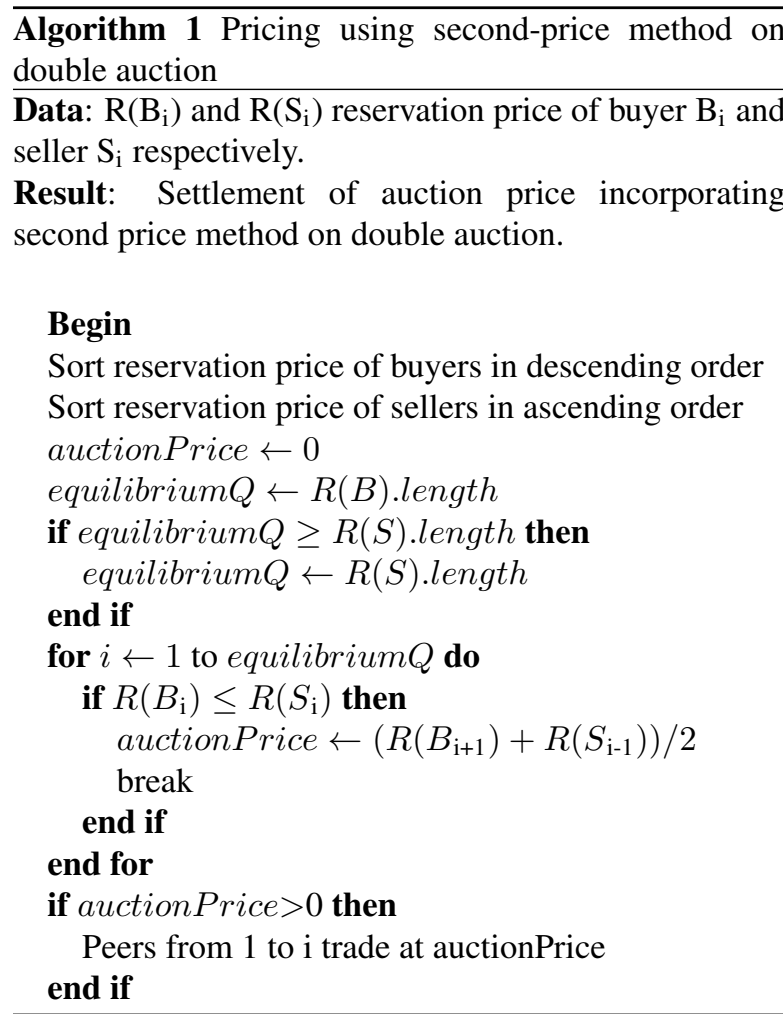

The description above shows the algorithmic steps of a system that incorporates second-price auction method into double auction method for pricing in a blockchain based distributed peer-to-peer energy trading. The smart contracts in the blockchain environment carry out these steps.

\subsection{Blockchain Framework}

Figure 7 shows the design of blocks and blockchain network in more detail, which also form a component of the proposed method. It shows unspent reservation profile, auction reservation data, auction data, and neighbourhood data and they are all stored in a blockchain ledger. We call this ledger as energy ledger. Smart contract conducts the auction, reads ledger, add new bids, finds neighbour, etc., upon request from peers within the network. Effectively, a smart contract updates the energy ledger. As this is a distributed system all peers in the network has access to the smart contract. In other words, the same set of rules or logics are available to all the peers across the peer-to-peer distributed double auction network.

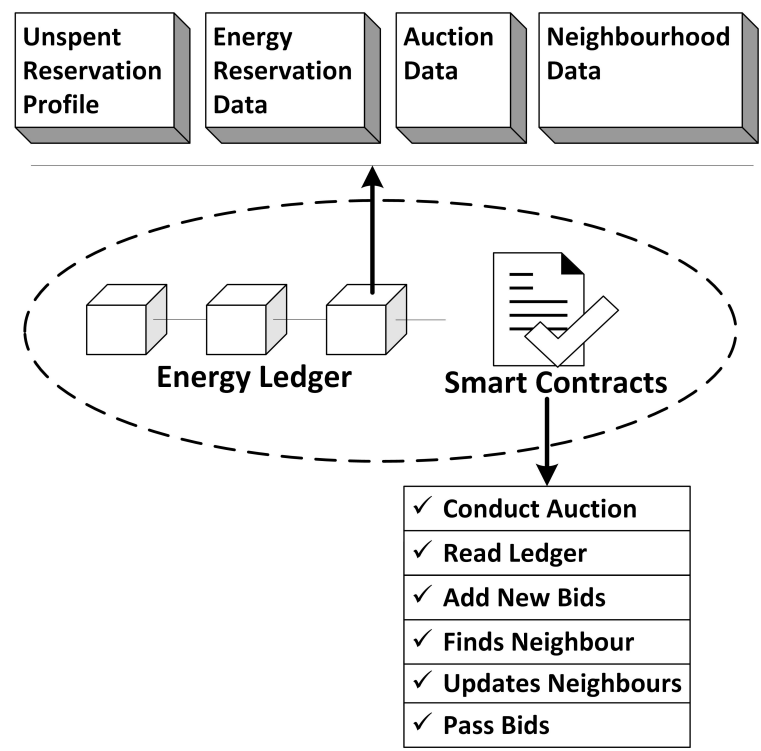

Figure 7. Blockchain design in Hyperledger Fabric for the proposed distributed double auction model

Thus, the blockchain design for the proposed distributed double auction model has these three features (a) all data required for peer-to-peer distributed double auction are stored in the ledger within Hyperledger Fabric. (b) the required set of rules for activities are stored in the form of programming script within a smart contract (c) the peers execute the smart contracts. 


\section{Experiment Setup}

To evaluate our proposed smart passing of unspent auction reservation and second-price auction in a distributed double auction, we simulated three peer-to-peer distributed double auction scenarios.

The first scenario is the traditional distributed double auction (DDA), which uses the equilibrium price as the auction price and conventional passing of unspent auction reservations developed by Thakur et al. [15]; this forms the baseline. The second scenario is unspent reservations with smart passing (URS1). The third scenario is unspent reservations with smart passing and second-price (URS2).

We consider three evaluation metrics to measure the performance of the three trading mechanisms:

i Convergence time indicated by the trail number to settle an auction.

ii Number of settled auctions in a 24 hours trading.

iii Utility of auction price data from successful reservations of all buyers.

The first two metrics measure the efficiency of a trading mechanism and the third metric measures the social welfare of the buyers.

\subsection{Environment}

The peer-to-peer double auction trading environment is simulated in Hyperledger Fabric, which provides facilities for setting up required peers, smart contracts and blockchain data ledgers. Hyperledger Fabric is installed within a Virtual Machine with 6.5 GB of RAM and 30 GB of disk space. Peers are established according to a varied requirement under a single organisation within Hyperledger Fabric. A smart contract in Hyperledger Fabric can be written in any of JavaScript, Java or Golang, but for our simulation, it is written in JavaScript. In Hyperledger Fabric, a smart contract can update a block but it maintains a history of all changes. Hyperledger Fabric is used as a blockchain platform: where all the peer houses and auctioneers are setup.

\subsection{Energy Data}

We used two major types of data in the experiments. The first data represents demand and supply data generated from 100 household meters with 5 minutes interval, courtesy of Thakur et al. [15]. The demand profiles data include 24 hours of demand records of 100 houses, and supply profiles data include photovoltaics data generated from the same houses throughout the day. From this data we take demand and supply profiles of the peak hours only. We consider 04:40 AM to 07:10 $\mathrm{PM}$ as peak time in our simulations. These data are converted to 15 minutes interval as meters in Australia give a reading in every 15 minutes. A meter, on behalf of a seller or buyer, is responsible for generating supply or demand data respectively. We assume that each meter generates $1 \mathrm{kWh}$ of supply or demand. The second type of data is the peer connection profile and the connectivity between peers are assigned randomly. Directly connected peers are immediate neighbours and they are physically close to each other.

Using the demand and supply as inputs, the simulation process generates three types of information: unspent reservation profile, auction reservation data and neighbour auctioneer data.

\subsection{Data Storage within Hyperledger Fabric}

Hyperledger Fabric facilitates data storage in blockchain ledger where the unit of data is a block and the smart contract is the interface to access that ledger. For our purpose of simulation, supply-demand data are updated through smart contract upon request of meters on behalf of peers. Peer connection profile in the ecosystem is loaded into the blockchain ledger as a block at the start of the simulation. Unspent reservation profile is a matrix and stored as another block in the ledger, which is updated when each unspent auction reservation is passed to an eligible auctioneer. Auction reservation data hold auction reservation price, trail number, auction price, etc. Each auction reservation resides in a block in the ledger. Similarly, the neighbour auctioneer data also resides in the ledger as a block. All data are updated through smart contract upon request from buyers or sellers in the ecosystem.

\section{Simulation and Results}

In this section we discuss and analyse the results of the simulation.

\subsection{Simulation}

We ran three different scenarios (DDA, URS1, and URS2) as described in Section 4 and measure their performance using the three metrics, which were also described in Section 4. In our experiments, we executed a set of all auctioneers asynchronously, assuming that all of them may have some energy reservations for further auction settlement. 
- We analyse the performance of the distributed double auction and smart passing of unspent auction reservation (URS1) by convergence time required to settle all auctions in peak hours. To evaluate convergence time from the simulation we collected trail numbers of successful energy auction reservations. For the comparison with URS1 we also simulated other approaches (DDA and URS2). As URS1 and URS2 include the smart passing of unspent auction reservation strategy, they will lead to better performance than DDA approach.

- We observe the auction throughput by the number of successful auctions processed by auctioneers with URS1 approach. We also simulate other approaches for comparison (DDA and URS2). As URS1 and URS2 include the smart passing of unspent auction reservation strategy, they will lead to more throughput than DDA approach.

- The utility of the buyers from all successful auction reservations (processed by URS2) are considered to measure the social welfare of buyers. Other approaches (DDA and URS1) are also simulated to compare the utility concerning social welfare. To analyse social welfare, we record auction settlement prices of successful auction reservations. Auction reservation prices are placed by buyers or sellers by keeping a range. For our experiment, the price range is $12 \dot{c}$ to $14 \dot{c}$. So, all the auction reservations made by all parties are kept between this range.

In the second-price auction method, the auction price is the second-highest price. When there is no second bidder then we use a bid floor of $10 \mathrm{c}$ as the second price. All three experiments use the same dataset so we can compare the performance from DDA, URS1, and URS2.

\subsection{Results}

We compare the performance of the three algorithms using three metrics: convergence time, number of successful auctions, and buyers utility (figures 8 to 10). Both URS1 and URS2 outperform DDA in all three metrics. URS1 and URS2 have similar convergence time and total number of successful auctions, but URS2 has better buyers' social welfare than URS1. We discuss the results in more detail below.

Figure 8 shows the efficiency of the three approaches by measuring the convergence time: both URS1 and URS2 converge earlier than the traditional double auction approach (DDA). All the auction reservations

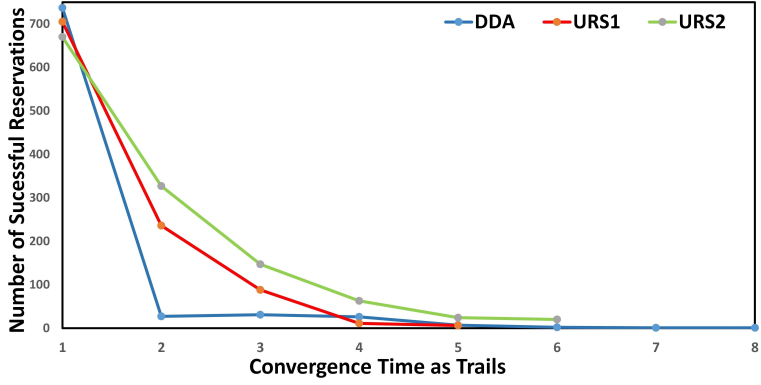

Figure 8. Convergence Time vs. Successful Energy Reservations for Three Approaches where DDA: distributed double auction approach, URS1: smart passing of unspent auction reservation with DDA, URS2: second-price auction with URS1

of URS1 converge within five trails, while for URS2 six trails. However, DDA takes a longer time (up to eight trails) to converge. It is also interesting to see that the convergence time between URS1 and URS2 are comparable, with URS2 being slightly better. As we are using the newly designed smart passing of unspent auction reservation approach in both URS1 and URS2, the unspent auction reservations are passed efficiently. Hence, the convergence time for these two approaches is better than DDA.

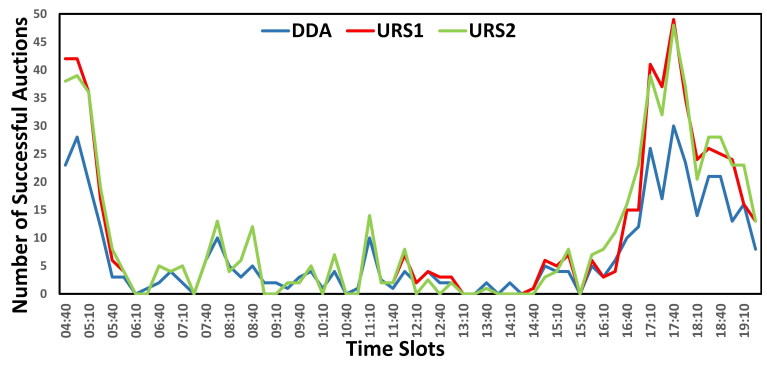

Figure 9. Number of Successful Auctions for Three Approaches

Figure 9 shows the efficiency of the performance of the three approaches concerning the total number of successful auctions of day-time trading. URS1 and URS2 settle higher number of auctions, whereas for each of the time slots DDA produces almost half of the auction compared to other two. While URS1 and URS2 are comparable, the URS2 processes the most number of auctions when combining the second-price auction method into the double auction method with unspent auction reservation approach. URS1 has slightly fewer number of successful auctions when using smart passing of unspent auction reservation approach and calculating the auction price using only double auction method. DDA performs the worst because it lacks smart passing 
of unspent auction reservation approach.

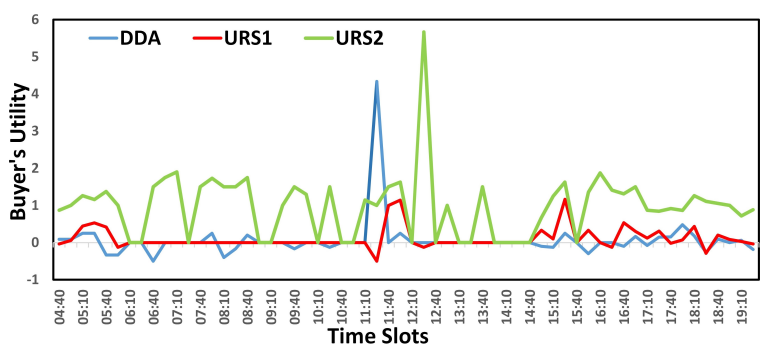

Figure 10. Comparison of Buyer's Utility for Three Approaches

Figure 10 shows the buyers' social welfare for the three approaches. To measure the social welfare of buyers, we used buyers' utility. The buyers' utility of DDA and URS1 are comparable: only these two methods use the equilibrium price as the auction price. On the other hand, URS2 generates almost four times of utility for buyers: it is the best performing method as it uses the second-price auction and distributed double auction with smart passing of unspent auction reservation approach.

From these experiments, it can be observed that a combination of double auction and smart passing of unspent auction reservation gives a superior auction convergence. This method can be further improved by using the second-price auction: together they offer a better utility and auction convergence time.

\section{Conclusion}

This paper presented a blockchain framework for implementing a novel energy trading mechanism. Auction performance and the social welfare of buyers are major concerns in this blockchain based distributed double auction. Passing unspent auction reservations to immediate neighbours affects the auction convergence which in turn affects the overall auction performance. On the other hand, buyers' utility becomes an essential factor for social welfare to attract more buyers in the auction process. As smart contracts and the blockchain environment reduce the cost of market settlement and enhance data immutability, a modern distributed auction market can use this trading mechanism in a blockchain based microgrid energy network ecosystem.

In this paper, we proposed a distributed double auction model that includes smart passing of unspent auction reservations to improve convergence time and the second-price auction method in a double auction method to improve buyers' utility. Experiment results show that in comparison with the existing distributed double auction method, our newly designed passing mechanism and pricing method gives superior performance in terms of auction convergence and buyers' utility. Design aspects of blockchain are intricate and pose new challenges that need to be addressed with further research.

One possible future extension of this work is to consider the price as another criterion to pass unspent reservations and to allow trading of variable energy quantity (not fixed at $1 \mathrm{kWh}$ ), which will lead to maximum number of energy quantity to be traded.

\section{Acknowledgement}

Md. Ezazul Islam is supported by the Henry Sutton PhD Scholarship stipend and Research Training Program (RTP) fee-offset scholarship through Federation University Australia.

\section{References}

[1] P. Thakkar, S. Nathan, and B. Viswanathan, "Performance benchmarking and optimizing hyperledger fabric blockchain platform," in 2018 IEEE 26th International Symposium on Modeling, Analysis, and Simulation of Computer and Telecommunication Systems (MASCOTS), pp. 264-276, IEEE, 2018.

[2] L. Hughes, Y. K. Dwivedi, S. K. Misra, N. P. Rana, V. Raghavan, and V. Akella, "Blockchain research, practice and policy: Applications, benefits, limitations, emerging research themes and research agenda," International Journal of Information Management, vol. 49, pp. 114-129, 2019.

[3] T. Alladi, V. Chamola, J. J. Rodrigues, and S. A. Kozlov, "Blockchain in smart grids: A review on different use cases," Sensors, vol. 19, no. 22, p. 4862, 2019.

[4] M. L. Di Silvestre, P. Gallo, J. M. Guerrero, R. Musca, E. R. Sanseverino, G. Sciumè, J. C. Vásquez, and G. Zizzo, "Blockchain for power systems: Current trends and future applications," Renewable and Sustainable Energy Reviews, vol. 119, p. 109585, 2020.

[5] H. Wu, J. Cao, Y. Yang, C. L. Tung, S. Jiang, B. Tang, Y. Liu, X. Wang, and Y. Deng, "Data management in supply chain using blockchain: Challenges and a case study," in 2019 28th International Conference on Computer Communication and Networks (ICCCN), pp. 1-8, IEEE, 2019.

[6] S. Perera, S. Nanayakkara, M. Rodrigo, S. Senaratne, and R. Weinand, "Blockchain technology: Is it hype or real in the construction industry?," Journal of Industrial Information Integration, vol. 17, p. 100125, 2020.

[7] V. Hassija, G. Bansal, V. Chamola, V. Saxena, and B. Sikdar, "Blockcom: A blockchain based commerce model for smart communities using auction mechanism," in 2019 IEEE International Conference on Communications Workshops (ICC Workshops), pp. 1-6, IEEE, 2019.

[8] S. Saxena, H. Farag, A. Brookson, H. Turesson, and H. Kim, "Design and field implementation of blockchain based renewable energy trading in residential communities," in 2019 2nd International Conference on Smart Grid and Renewable Energy (SGRE), pp. 1-6, IEEE, 2019. 
[9] J. Guerrero, A. C. Chapman, and G. Verbič, "Decentralized p2p energy trading under network constraints in a low-voltage network," IEEE Transactions on Smart Grid, vol. 10, no. 5, pp. 5163-5173, 2018.

[10] D. Tse, B. Zhang, Y. Yang, C. Cheng, and H. Mu, "Blockchain application in food supply information security," in 2017 IEEE international conference on industrial engineering and engineering management (IEEM), pp. 1357-1361, IEEE, 2017.

[11] A. Mentsiev, E. Guzueva, S. Yunaeva, M. Engel, and M. Abubakarov, "Blockchain as a technology for the transition to a new digital economy," in Journal of Physics: Conference Series, vol. 1399, p. 033113, IOP Publishing, 2019.

[12] M. Andoni, V. Robu, D. Flynn, S. Abram, D. Geach, D. Jenkins, P. McCallum, and A. Peacock, "Blockchain technology in the energy sector: A systematic review of challenges and opportunities," Renewable and Sustainable Energy Reviews, vol. 100, pp. 143-174, 2019.

[13] J. Wu and N. K. Tran, "Application of blockchain technology in sustainable energy systems: An overview," Sustainability, vol. 10, no. 9, p. 3067, 2018.

[14] J. Lin, M. Pipattanasomporn, and S. Rahman, "Comparative analysis of auction mechanisms and bidding strategies for $\mathrm{p} 2 \mathrm{p}$ solar transactive energy markets," Applied energy, vol. 255, p. 113687, 2019.

[15] S. Thakur, B. P. Hayes, and J. G. Breslin, "Distributed double auction for peer to peer energy trade using blockchains," in 2018 5th International Symposium on Environment-Friendly Energies and Applications (EFEA), pp. 1-8, IEEE, 2018.

[16] C. Cachin et al., "Architecture of the hyperledger blockchain fabric," in Workshop on distributed cryptocurrencies and consensus ledgers, vol. 310, Chicago, IL, 2016.

[17] S. Bikhchandani, "Reputation in repeated second-price auctions," Journal of Economic Theory, vol. 46, no. 1, pp. 97-119, 1988 .

[18] K. Omote and A. Miyaji, "A second-price sealed-bid auction with verifiable discriminant of p 0-th root," in International Conference on Financial Cryptography, pp. 57-71, Springer, 2002.

[19] R. P. McAfee, "A dominant strategy double auction," Journal of economic Theory, vol. 56, no. 2, pp. 434-450, 1992.

[20] B. R. Chen and Y. S. Chiu, "Competitive bidding with a bid floor," International Journal of Economic Theory, vol. 7, no. 4, pp. 351-371, 2011.

[21] W. Tushar, T. K. Saha, C. Yuen, D. Smith, and H. V. Poor, "Peer-to-peer trading in electricity networks: An overview," IEEE Transactions on Smart Grid, vol. 11, no. 4, pp. 3185-3200, 2020.

[22] Z. Zhao, K. Nakayama, and R. Sharma, "Decentralized transactive energy auctions with bandit learning," in 2019 IEEE PES Transactive Energy Systems Conference (TESC), pp. 1-5, IEEE, 2019.

[23] X. Zou, "Double-sided auction mechanism design in electricity based on maximizing social welfare," Energy Policy, vol. 37, no. 11, pp. 4231-4239, 2009.
[24] B. P. Majumder, M. N. Faqiry, S. Das, and A. Pahwa, "An efficient iterative double auction for energy trading in microgrids," in 2014 IEEE Symposium on Computational Intelligence Applications in Smart Grid (CIASG), pp. 1-7, IEEE, 2014. 